\title{
D-brane-anti-D-brane effective action and brane interaction in open string channel
}

\author{
Mohammad R. Garousi \\ Department of Physics, Ferdowsi university, P.O. Box 1436, Mashhad, Iran \\ Institute for Studies in Theoretical Physics and Mathematics IPM \\ P.O. Box 19395-5531, Tehran, Iran
}

\begin{abstract}
We construct the effective action of a $D_{p}$-brane-anti- $D_{p}$-brane system by making use of the non-abelian extension of tachyonic DBI action. We succeed the construction by restricting the Chan-Paton factors of two non-BPS $D_{p}$-branes in the action to the Chan-Paton factors of a $D_{p} \bar{D}_{p}$ system. For the special case that both branes are coincident, the action reduces to the one proposed by A. Sen.

The effective $D_{p} \bar{D}_{p}$ potential indicates that when branes separation is larger than the string length scale, there are two minima in the tachyon direction. As branes move toward each other under the gravitational force, the tachyon tunneling from false to true vacuum may make a bubble formation followed by a classical evolution of the bubble. On the other hand, when branes separation is smaller than the string length scale, the potential shows one maximum and one minimum. In this case, a homogeneous tachyon rolling in real time makes an attractive potential for the branes distance. This classical force is speculated to be the effective force between the two branes.
\end{abstract}




\section{The idea}

Study of unstable objects in string theory might shed new light in understanding properties of string theory in time-dependent backgrounds [1, 2, 3, 4, 5, 6. Generally speaking, source of instability in these processes is appearance of some tachyonic modes in the spectrum of these unstable objects. It then makes sense to study these objects in a field theory which includes those modes. In this regard, it has been shown by A. Sen that an effective action of Born-Infeld type proposed in [7, 8, 9, 10] can capture many properties of the decay of non-BPS $\mathrm{D}_{p}$-branes in string theory [2, 3]. Having an effective action, one may then study evolution of these unstable objects in time-dependent backgrounds. See [1] for possible cosmological application of the tachyonic DBI action.

Another unstable object in string theory is parallel $\mathrm{D}_{p}$-brane anti- $\mathrm{D}_{p}$-brane system (see e.g., , [12]). Detailed study of this object reveals when brane separation is smaller than the string length scale, spectrum of this system has two tachyonic modes [13]. These modes however become massive when brane separation is larger than the string length scale. The effective action should then depend on brane separation. When it is smaller than the string length scale, the effective action for dynamics and for decay of branes should include the tachyonic modes because these are the most important modes which rule the evolution of the system. On the other hand, when brane separation is larger than the string length scale, the effective action which is a low energy effective action, should be in terms of massless closed string fields propagating between the two branes. This action describes properly the dynamics of the branes, however, it is not an appropriate action for studying decay of the whole system, i.e., bubble formation followed by a classical evolution of the bubble. This bubble formation should appear in an effective action which includes false and true vacuums.

In the literature, there are some proposal for $D_{p} \bar{D}_{p}$ effective action when branes are coincident [14, 15. On the other hand, when brane separation is larger than the string length scale, the low energy gravity effective action is the Coulomb attraction force due to gravity and RR fields. Making use of this latter effective action, the cosmology of $D_{3} \bar{D}_{3}$ system has been discussed in [16].

In this paper, for arbitrary branes separation, we would like to present an effective action for parallel $D_{p} \bar{D}_{p}$ system in terms of transverse scalars and tachyonic fields. We speculate this effective action should describe the dynamics and the decay of system when branes separation is smaller than the string length scale, and should describe the decay of the system when branes separation is larger than the string length scale.

Our method for finding this effective action is as follows. One may try to extract the effective action from the string theory S-matrix elements, like what has been done for nonBPS $D_{p}$-brane case [8, 17]. The tree level world sheet is disk and various vertex operators are the same as in the non-BPS $D_{p}$-brane case. The only difference is that now one has to take 
appropriate Chan-Paton factors into account. On the other hand, the Chan-Paton factors of $D_{p} \bar{D}_{p}$ system are some subgroup of the Chan-Paton factors of two non-BPS $D_{p}$-branes, and the effective action of two non-BPS $D_{p}$-branes consistent with string theory S-matrix elements is given by the nonabelian extension of tachyonic DBI action [8]. Restricting the Chan-Paton factors of two non-BPS $D_{p}$-branes in the nonabelian tachyonic DBI action to the Chan-Paton factors of a $D_{p} \bar{D}_{p}$ system, we shall find effective action of the parallel $D_{p} \bar{D}_{p}$ system $^{1}$

In the next section we will find the effective action of the $D_{p} \bar{D}_{p}$ system by constraining the Chan-Paton factors of different open string fields in the non-abelian tachyonic DBI action [8]. The action includes coupling to gravity background and world-volume gauge fields. In section 3, we simplify the action to include only the real part of tachyon and the relative distance of the branes. Using this action, we then discuss the dynamics and the decay of the branes.

\section{$2 \quad D_{p} \bar{D}_{p}$ effective action}

The proposed effective action for describing the decay and the dynamics of a non-BPS $\mathrm{D}_{p}$-brane, and its coupling to gravity and world-volume gauge field is given by [8, 9, 10]:

$$
S=-\int d^{p+1} \sigma V(T) e^{-\Phi} \sqrt{-\operatorname{det}\left(P\left[g_{a b}+B_{a b}\right]+2 \pi \alpha^{\prime} F_{a b}+2 \pi \alpha^{\prime} \partial_{a} T \partial_{b} T\right)},
$$

where $V(T)=T_{p}\left(1-\frac{\pi}{2} T^{2}+O\left(T^{4}\right)\right)$ is the tachyon potential. Here $g_{a b}, B_{a b}, \Phi$ and $A_{a}$ are the spacetime metric, antisymmetric Kalb-Ramond tensor, dilaton and the gauge field, respectively. In above action $P[\cdots]$ is also the pull-back of the closed string fields. For example, $P\left[\eta_{a b}\right]=\eta_{\mu \nu} \partial_{a} X^{\mu} \partial_{b} X^{\nu}=\eta_{a b}+\partial_{a} X^{i} \partial_{b} X_{i}$ in the static gauge ${ }^{2}$.

The kink solution of the equation of motion of tachyon should be the BPS $\mathrm{D}_{p-1}$-brane 22. The tension of the kink is given by $T_{p-1}=\sqrt{2 \pi \alpha^{\prime}} \int_{-T_{0}}^{T_{0}} V(T) d T$ where $T_{0}$ is the value of the tachyon potential at its minimum. There are many different tachyon potentials which correctly reproduce the tension of the BPS brane [10, 14, 23], i.e., $T_{p-1}=\pi \sqrt{2 \alpha^{\prime}} T_{p}$. One example is the following potential [24, 5]:

$$
V(T)=\frac{T_{p}}{\cosh (\sqrt{\pi} T)}
$$

\footnotetext{
${ }^{1}$ The restriction of Chan-Paton factors may result from orbifolding type II string theory 12. As pointed out in [12, the moding out one non-BPS $D_{p}$-brane in type IIA/IIB by $(-1)^{F_{L}^{S}}$ has a two-fold ambiguity. It may end up either with a $D_{p}$-brane or a $\bar{D}_{p}$-brane in type IIB/IIA. This gives a four-fold ambiguity for moding out two non-BPS $D_{p}$-branes by $(-1)^{F_{L}^{S}}$. It may end up with a $D_{p} D_{p}$, a $\bar{D}_{p} D_{p}$, a $D_{p} \bar{D}_{p}$, or a $\bar{D}_{p} \bar{D}_{p}$ system. In general, there should be a $2^{N}$-fold ambiguity for moding out $N$ non-BPS $D_{p}$-branes by $(-1)^{F_{L}^{S}}$.

${ }^{2}$ Our index convention is that $\mu, \nu, \ldots=0,1, \ldots, 9 ; a, b, \ldots=0,1, \ldots, p$ and $i, j, \ldots=p+1, \ldots, 9$.
} 
This has minimum at $T \rightarrow \pm \infty$ and behaves as $V(T) \sim e^{-\sqrt{\pi} T}$ at $T \rightarrow \infty$. This potential is also consistent with the fact that there is no open string state at the end of the tachyon condensation [3].

Now consider N non-BPS D-branes. They should be described effectively by non-abelian extension of the above action which is $[8]^{3}$

$$
\begin{aligned}
S= & -\int d^{p+1} \sigma \operatorname{Tr}\left(V(T) \sqrt{\operatorname{det}\left(Q^{i}{ }_{j}\right)}\right. \\
& \left.\times e^{-\Phi(X)} \sqrt{-\operatorname{det}\left(P\left[E_{a b}(X)+E_{a i}(X)\left(Q^{-1}-\delta\right)^{i j} E_{j b}(X)\right]+2 \pi \alpha^{\prime} F_{a b}+T_{a b}\right)}\right),
\end{aligned}
$$

where $E_{\mu \nu}=g_{\mu \nu}+B_{\mu \nu}$. The indices in this action are raised and lowered by $E^{i j}$ and $E_{i j}$, respectively. The matrices $Q^{i}{ }_{j}$ and $T_{a b}$ are

$$
\begin{aligned}
Q_{j}^{i}= & I \delta^{i}{ }_{j}-\frac{i}{2 \pi \alpha^{\prime}}\left[X^{i}, X^{k}\right] E_{k j}(X)-\frac{1}{2 \pi \alpha^{\prime}}\left[X^{i}, T\right]\left[X^{k}, T\right] E_{k j}(X), \\
T_{a b}= & 2 \pi \alpha^{\prime} D_{a} T D_{b} T+D_{a} T\left[X^{i}, T\right]\left(Q^{-1}\right)_{i j}\left[X^{j}, T\right] D_{b} T \\
& +i E_{a i}(X)\left(Q^{-1}\right)^{i}{ }_{j}\left[X^{j}, T\right] D_{b} T+i D_{a} T\left[X^{i}, T\right]\left(Q^{-1}\right)_{i}{ }^{j} E_{j b}(X) \\
& +i D_{a} X^{i}\left(Q^{-1}\right)_{i j}\left[X^{j}, T\right] D_{b} T-i D_{a} T\left[X^{i}, T\right]\left(Q^{-1}\right)_{i j} D_{b} X^{j}
\end{aligned}
$$

The trace in the action (3) should be completely symmetric between all non-abelian expressions of the form $F_{a b}, D_{a} X^{i},\left[X^{i}, X^{j}\right], D_{a} T,\left[X^{i}, T\right]$, individual $T$ of the tachyon potential and individual $X^{i}$ of the Taylor expansion of the closed string fields in the action[26]. The pull-back of closed string fields is defined in the static gauge in which the derivatives are covariant derivative, e.g., , $P\left[\eta_{a b}\right]=\eta_{a b}+D_{a} X^{i} D_{b} X^{j} \eta_{i j}$. The gauge field strength and covariant derivative of transverse scalars and tachyons are

$$
\begin{aligned}
F_{a b} & =\partial_{a} A_{b}-\partial_{b} A_{a}-i\left[A_{a}, A_{b}\right], \\
D_{a} X^{i} & =\partial_{a} X^{i}-i\left[A_{a}, X^{i}\right], \\
D_{a} T & =\partial_{a} T-i\left[A_{a}, T\right] .
\end{aligned}
$$

Obviously the action (3) has $U(N)$ gauge symmetry when all branes are coincident. This is resulted from the fact that all gauge fields corresponding to the open string stretched between branes are massless in this case. When all branes are separated in the transverse space, only the gauge fields corresponding to the open strings with both ends on one brane remain massless. In this case the $U(N)$ symmetry breaks to $U(1)^{N}$ symmetry.

One may confirm various couplings in the action (3) by studying appropriate disk level S-matrix elements in string theory [8, 17]. From S-matrix elements point of view, there is no difference between calculation of various fields coupling on two non-BPS D-brane and on D-brane anti-D-brane. Both involve exactly the same calculation. However, the difference

\footnotetext{
${ }^{3}$ See [25], for the non-abelian extension of Chern-Simons term for non-BPS D-branes.
} 
is only on the choice of Chan-Paton factors. For two non-BPS D-branes, the Chan-Paton factors are such that the open string fields are

$$
A_{a}=\left(\begin{array}{cc}
A_{a}^{(11)} & A_{a}^{(12)} \\
A_{a}^{(21)} & A_{a}^{(22)}
\end{array}\right), X^{i}=\left(\begin{array}{ll}
X^{(11) i} & X^{(12) i} \\
X^{(21) i} & X^{(22) i}
\end{array}\right), T=\left(\begin{array}{cc}
T^{(11)} & T^{(12)} \\
T^{(21)} & T^{(22)}
\end{array}\right)
$$

where superscripts $(11),(12),(21),(22)$ refers to the end of open strings, e.g., (12) means the open string with one end on brane 1 and the other end on brane 2. As we mentioned above, the symmetry of the theory depends on the masses of $A_{a}^{(12)}$ and $A_{a}^{(21)}$ which can be calculated from the term $\operatorname{Tr}\left(D_{a} X^{i} D^{a} X_{i}\right)$ in the action (3). They are massless for coincident branes hence, the symmetry is $U(2)$, and they are massive for non-coincident branes hence, the symmetry is $U(1) \times U(1)$.

For D-brane anti-D-brane system, the matrices $A_{a}, X^{i}$ and $T$ are

$$
A_{a}=\left(\begin{array}{cc}
A_{a}^{(1)} & 0 \\
0 & A_{a}^{(2)}
\end{array}\right), X^{i}=\left(\begin{array}{cc}
X^{(1) i} & 0 \\
0 & X^{(2) i}
\end{array}\right), T=\left(\begin{array}{cc}
0 & \tau \\
\tau^{*} & 0
\end{array}\right) .
$$

We have changed the notation. The superscripts (1) and (2) refer to the open string fields with both ends on brane 1 and 2, respectively. $\tau\left(\tau^{*}\right)$ refers to the tachyon with one end on brane 1(2) and the other end on brane 2(1). Since there is no off-diagonal terms for the gauge field, the theory has gauge symmetry $U(1) \times U(1)$ independent of the position of branes. The above matrices satisfy the following relations:

$$
\begin{gathered}
{\left[X^{i}, X^{j}\right]=0, \quad\left[X^{i}, A_{a}\right]=0, \quad\left[A_{a}, A_{b}\right]=0} \\
{\left[X^{i}, T\right]=\ell^{i}\left(\begin{array}{cc}
0 & \tau \\
-\tau^{*} & 0
\end{array}\right),\left[A_{a}, T\right]=\left(A_{a}^{(1)}-A_{a}^{(2)}\right)\left(\begin{array}{cc}
0 & \tau \\
-\tau^{*} & 0
\end{array}\right),}
\end{gathered}
$$

where $\ell^{i}=X^{(1) i}-X^{(2) i}$ is the distance between the two branes. Hence, the non-abelian gauge field strength and covariant derivative of the transverse scalars become abelian, i.e., $F_{a b}=\partial_{a} A_{b}-\partial_{b} A_{a}$ and $D_{a} X^{i}=\partial_{a} X^{i}$. The matrix $Q^{i}{ }_{j}$ in (4) simplifies to

$$
Q_{j}^{i}=\left(I \delta^{i}{ }_{j}+\frac{|\tau|^{2}}{2 \pi \alpha^{\prime}} \ell^{i} \ell^{k} E_{k j}(X)\right) .
$$

The inverse of this matrix is

$$
\left(Q^{-1}\right)_{j}^{i}=\left(I \delta^{i}{ }_{j}-\frac{|\tau|^{2}}{\left(2 \pi \alpha^{\prime}\right) \operatorname{det}(Q)} \ell^{i} \ell^{k} E_{k j}(X)\right)
$$

where

$$
\operatorname{det}(Q)=\left(I+\frac{|\tau|^{2}}{2 \pi \alpha^{\prime}} \ell^{i} \ell^{k} g_{k i}(X)\right)
$$


It is easy to check that $Q^{i}{ }_{j}\left(Q^{-1}\right)_{k}^{j}=I \delta_{k}^{i}$. The matrix $T_{a b}$ in (44) simplifies to

$$
\begin{aligned}
T_{a b}= & (\operatorname{det}(Q))^{-1}\left(2 \pi \alpha^{\prime} D_{a} T D_{b} T+i\left(E_{a i}(X)+\partial_{a} X^{j} E_{j i}(X)\right) \ell^{i}\left(\begin{array}{cc}
0 & \tau \\
-\tau^{*} & 0
\end{array}\right) D_{b} T\right. \\
& \left.+i D_{a} T\left(\begin{array}{cc}
0 & \tau \\
-\tau^{*} & 0
\end{array}\right) \ell^{i}\left(E_{i b}(X)-E_{i j}(X) \partial_{b} X^{j}\right)\right)
\end{aligned}
$$

Note that this matrix is not a real matrix, however, one expects to have a real action after implementing the trace prescription.

Now using these facts that the trace in the action should be symmetric, $F_{a b}, X^{i}, T^{2}$ are diagonal matrices and $D_{a} T,\left[X^{i}, T\right]$ are off-diagonal matrices, one can implement the symmetric trace by writing $D_{a} T D_{b} T$ and $\left[X^{i}, T\right] D_{a} T$ in $T_{a b}$ in a symmetric form. That is, $D_{a} T D_{b} T \rightarrow\left(D_{a} T D_{b} T+D_{b} T D_{a} T\right) / 2$ and $\left[X^{i}, T\right] D_{a} T \rightarrow\left(\left[X^{i}, T\right] D_{a} T+D_{a} T\left[X^{i}, T\right]\right) / 2$. Hence, $T_{a b}$ becomes

$$
\begin{aligned}
T_{a b}= & (\operatorname{det}(Q))^{-1}\left(\frac{I 2 \pi \alpha^{\prime}}{2}\left(D_{a} \tau\left(D_{b} \tau\right)^{*}+D_{b} \tau\left(D_{a} \tau\right)^{*}\right)\right. \\
& +\frac{i}{2}\left(E_{a i}(X)+\partial_{a} X^{j} E_{j i}(X)\right) \ell^{i}\left(\tau\left(D_{b} \tau\right)^{*}-\tau^{*} D_{b} \tau\right) \\
& \left.+\frac{i}{2}\left(\tau\left(D_{a} \tau\right)^{*}-\tau^{*} D_{a} \tau\right) \ell^{i}\left(E_{i b}(X)-E_{i j}(X) \partial_{b} X^{j}\right)\right),
\end{aligned}
$$

where the covariant derivative of tachyon is $D_{a} \tau=\partial_{a} \tau-i\left(A_{a}^{(1)}-A_{a}^{(2)}\right) \tau$. As anticipated above, the matrix $T_{a b}$ is now real.

Now inserting the above expression in (3) and performing the trace, one finds the following action for $D_{p} \bar{D}_{p}$ system in arbitrary background at tree level:

$$
S=-\int d^{p+1} \sigma\left(\mathcal{V}^{(1)}(|\tau|, \ell) e^{-\Phi\left(X^{(1)}\right)} \sqrt{-\operatorname{det} \mathbf{A}^{(1)}}+\mathcal{V}^{(2)}(|\tau|, \ell) e^{-\Phi\left(X^{(2)}\right)} \sqrt{-\operatorname{det} \mathbf{A}^{(2)}}\right)
$$

where

$$
\begin{aligned}
\mathbf{A}_{a b}^{(n)}= & P^{(n)}\left[E_{a b}\left(X^{(n)}\right)-\frac{|\tau|^{2}}{2 \pi \alpha^{\prime} \operatorname{det}\left(Q^{(n)}\right)} E_{a i}\left(X^{(n)}\right) \ell^{i} \ell^{j} E_{j b}\left(X^{(n)}\right)\right]+2 \pi \alpha^{\prime} F_{a b}^{(n)} \\
& +\frac{1}{\operatorname{det}\left(Q^{(n)}\right)}\left(\frac{2 \pi \alpha^{\prime}}{2}\left(D_{a} \tau\left(D_{b} \tau\right)^{*}+D_{b} \tau\left(D_{a} \tau\right)^{*}\right)\right. \\
& +\frac{i}{2}\left(E_{a i}\left(X^{(n)}\right)+\partial_{a} X^{(n) j} E_{j i}\left(X^{(n)}\right)\right) \ell^{i}\left(\tau\left(D_{b} \tau\right)^{*}-\tau^{*} D_{b} \tau\right) \\
& \left.+\frac{i}{2}\left(\tau\left(D_{a} \tau\right)^{*}-\tau^{*} D_{a} \tau\right) \ell^{i}\left(E_{i b}\left(X^{(n)}\right)-E_{i j}\left(X^{(n)}\right) \partial_{b} X^{(n) j}\right)\right)
\end{aligned}
$$

where $n=1,2$. In the above equation $P^{(n)}[\ldots]$ means pull-back of closed string fields on the $n$-th brane, e.g., $P^{(1)}\left[\eta_{a b}\right]=\eta_{a b}+\partial_{a} X_{i}^{(1)} \partial_{b} X_{j}^{(1)} \eta^{i j}$. The $D \bar{D}$ potential is

$$
\mathcal{V}^{(n)}(|\tau|, \ell)=V(|\tau|) \sqrt{\operatorname{det}\left(Q^{(n)}\right)}
$$




$$
=V(|\tau|) \sqrt{1+\frac{|\tau|^{2}}{2 \pi \alpha^{\prime}} \ell^{i} \ell^{k} g_{k i}\left(X^{(n)}\right)}
$$

where $V(|\tau|)$ is the tachyon potential of non-BPS D-brane. For small $|\tau|$ it has the expansion

$$
\mathcal{V}^{(n)}(|\tau|, \ell)=T_{p}\left(1+\frac{2 \pi \alpha^{\prime}}{2}\left(\frac{\ell^{i} \ell^{j} g_{i j}\left(X^{(n)}\right)}{\left(2 \pi \alpha^{\prime}\right)^{2}}-\frac{1}{2 \alpha^{\prime}}\right)|\tau|^{2}+\mathcal{O}\left(|\tau|^{4}\right)\right) .
$$

The second term in the second parentheses above is the mass squared of the tachyon and the first term is the mass squared of the string stretched between two branes, i.e., (tension $)^{2} \times(\text { length })^{2}$. Note that potential had local minimum at $|\tau|=0$ only when $\ell>\sqrt{2 \pi^{2} \alpha^{\prime}}$.

For trivial closed string background and for coincident branes, $\ell=0$, the action (13) simplifies to

$$
S=-\int d^{p+1} \sigma V(|\tau|)\left(\sqrt{-\operatorname{det} \mathbf{A}^{(1)}}+\sqrt{-\operatorname{det} \mathbf{A}^{(2)}}\right)
$$

where

$$
\mathbf{A}_{a b}^{(n)}=\eta_{a b}+\partial_{a} X_{i}^{(n)} \partial_{b} X_{j}^{(n)} \eta^{i j}+2 \pi \alpha^{\prime} F_{a b}^{(n)}+\frac{2 \pi \alpha^{\prime}}{2}\left(D_{a} \tau\left(D_{b} \tau\right)^{*}+D_{b} \tau\left(D_{a} \tau\right)^{*}\right)
$$

The action (17) is the one proposed in [14] when two branes are coincident.

\section{$3 D_{p} \bar{D}_{p}$ interaction in open string channel}

In string theory, the interaction between two D-branes with the same RR charge is given by zero point function on cylindrical world-sheet [18]. This world-sheet has two dual descriptions. In terms of closed strings or in terms of open strings. In the former which is a classical process, a closed string is created by one brane. It propagates in the transverse space between the two branes, and then the other brane absorbs it. In the latter which is a quantum process, a pair of open strings stretching between the two branes are created by vacuum. They propagate and then annihilate back to the vacuum. The whole amplitude is zero due to an identity.

This interaction can also be studied in low energy effective field theories. If branes are far from each other, the only massless fields are the graviton multiplete. So the effective action is in terms of gravity. That is to say, one of the branes produces gravitational field due to its mass and RR charge, and the other brane moves in this background as a probe. The repulsive force of $\mathrm{RR}$ charge cancels out the attractive force of their mass. So the net classical force is zero. On the other hand, when both branes are coincident, the lowest mode of the open string stretching between the two branes and the graviton multiplete are both 
massless. So the low energy interaction can be either in terms of closed string or in terms of open string fields. In the closed string channel, again the RR and gravity forces cancel each other. In the open string channel, the effective field theory includes massless bosons and fermions. The positive one loop vacuum energy of bosons cancels out the negative vacuum energy of the fermions. Then, there is no quantum interaction between the two branes which is consistent with the gravity channel.

In string theory, when brane separation is larger than the string length scale, interaction between a $D_{p}$-brane and a $\bar{D}_{p}$-brane is again given by zero point function on cylindrical world-sheet [18]. This world-sheet has again two dual descriptions as in the $D_{p} D_{p}$ system. However, unlike the $D_{p} D_{p}$ system, there is no identity to make the whole amplitude to be zero. This interaction can also be studied in a low energy effective field theory. The only massless fields are the graviton multiplete. So the low energy effective action is in the closed string channel. Unlike the previous case, however, the repulsive force of RR charge dose not cancel out the attractive force of their mass. So the net classical force is non-zero, in accord with string theory result. Note that the separated $D_{p} \bar{D}_{p}$ system is unstable, however, the decay mechanism is not described by the above world-sheet nor by the above low energy effective action.

On the other side, when brane separation is smaller than the string length scale, interaction of a $D_{p}$-brane and a $\bar{D}_{p}$-brane in terms of cylindrical world-sheet gives a complex force [21]. This indicates there is a tachyonic mode for the open string stretching between the two branes. Moreover, it indicates that the force between the two branes in full string theory is not given by the above simple world-sheet any more. To follow the system in effective theory, one needs an effective potential which includes all order of tachyon fields. Making use of the proposed effective action of (13), we would like to discuss in this section the dynamics and the decay of the system when brane separation is smaller than the string length scale, and the decay of the system when brane separation is larger than the string scale.

To simplify the discussion, we consider trivial background and consider the case where branes are separated in the $k$-th transverse direction. Writing $\tau=\phi e^{i \theta}$ and considering homogeneous case, action (13) simplifies to

$$
S=-\int d^{p+1} \sigma \mathcal{V}(\phi, \ell)\left(\sqrt{-\mathbf{A}_{00}^{(1)}}+\sqrt{-\mathbf{A}_{00}^{(2)}}\right)
$$

where

$$
\begin{aligned}
\mathcal{V}(\phi, \ell) & =V(\phi) \sqrt{1+\frac{\phi^{2} \ell^{2}}{2 \pi \alpha^{\prime}}} \\
\mathbf{A}_{00}^{(n)} & =-1+\dot{X}^{(n) i} \dot{X}^{(n) j} \eta_{i j}+\frac{1}{1+\frac{\phi^{2} \ell^{2}}{2 \pi \alpha^{\prime}}}\left(\dot{X}^{(n) k} \dot{X}^{(n) k}+2 \pi \alpha^{\prime}\left(\dot{\phi}^{2}+\phi^{2} \dot{\theta}^{2}\right)\right)
\end{aligned}
$$

where $i, j \neq k$. Furthermore, writing $X^{(n) k}$ in terms of center of mass, $R$, and in terms of brane separation, $\ell$, i.e., $X^{(1) k}=R+\ell / 2$ and $X^{(2) k}=R-\ell / 2$, one finds that the action has 
no potential for $R, X^{(n) i}$ nor for $\theta$. So their equations of motion are satisfied for constant $R, X^{(n) i}$ and $\theta$. This simplifies the above action to

$$
S=-2 \int d^{p+1} \sigma \mathcal{V}(\phi, \ell) \sqrt{1-\frac{1}{1+\frac{\phi^{2} \ell^{2}}{2 \pi \alpha^{\prime}}}\left(\frac{1}{4} \dot{\ell}^{2}+2 \pi \alpha^{\prime} \dot{\phi}^{2}\right)} .
$$

Note that for $D_{p} D_{p}$ system, the corresponding action is $S=-2 T_{p} \int d^{p+1} \sigma \sqrt{1-\dot{\ell}^{2} / 4}$ which gives no potential for $\ell$. The effective force in this case is the attractive Coulomb force due to the tension of the branes, though, this force is canceled by the contribution from the repulsive $\mathrm{RR}$ force.

The effective potential (20) along the $\ell$ direction has a minimum at $\ell=0$ for any nonzero $\phi$. Because the tachyon potential (2) goes to zero as $e^{-\sqrt{\pi} \phi}$, the effective potential $\mathcal{V}(\phi, \ell)$ along the $\phi$ direction has two minima for $\ell>\sqrt{2 \pi^{2} \alpha^{\prime}}$. One at $\phi=0$ and the other one at $\phi \rightarrow \infty$. On the other hand, for $\ell<\sqrt{2 \pi^{2} \alpha^{\prime}}$, it has one maximum and one minimum (see Fig1). Actually, these behaviours of the effective potential $\mathcal{V}(\phi, \ell)$ are independent of the form of tachyon potential $V(\phi)$, so long as the tachyon potential goes to zero asymptotically faster than $1 / \phi$.

As an initial condition, consider a non-coincident stationary $D_{p} \bar{D}_{p}$ system with a coherent quantum fluctuation for tachyon, i.e., $\phi=\epsilon$. The effective potential for this initial condition is $\mathcal{V}(\epsilon, \ell) \simeq T_{p}$. If $\ell<\sqrt{2 \pi^{2} \alpha^{\prime}}$, the tachyon can roll down the potential in real time. This non-zero tachyon generates a time dependent force for $\ell$. If $\phi_{c l}(t, \ell)$ is the homogeneous tachyon rolling solution of the above action, the potential in the $\ell$ direction becomes,

$$
\mathcal{V}\left(\phi_{c l}, \ell\right)=V\left(\phi_{c l}(t, \ell)\right) \sqrt{1+\frac{\phi_{c l}^{2}(t, \ell) \ell^{2}}{2 \pi \alpha^{\prime}}}
$$

which produces a non-zero force. We speculate that this homogeneous classical force might be the effective force between the two branes.

On the other hand, if initial value of $\ell$ is $\ell>\sqrt{2 \pi^{2} \alpha^{\prime}}$, there is no real time homogeneous tachyon solution that penetrates the barrier of the tachyon potential. This is consistent with the fact that the effective force for dynamics of the branes in this case is in the closed string channel, i.e., the homogeneous Coulomb force. However, the decay of the branes should be in the open string channel. In fact the effective potential in Fig.1 indicates that there is an instanton effect where system will tunnel out of the false vacuum. There should be an inhomogeneous "bounce" solution to the effective action [27]. This bounce solution should form a bubble inside which the tachyon is in the true vacuum and outside which the tachyon is in the false vacuum. The solution should also include a throat formation in the $\ell$ direction [19]. The classical evolution of the tachyon after penetrating through the potential barrier, should make the bubble/throat to be expanded to infinity leaving behind 


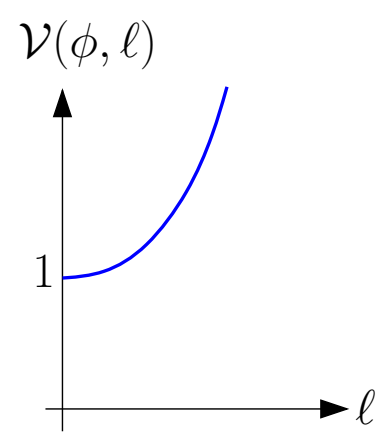

(a)

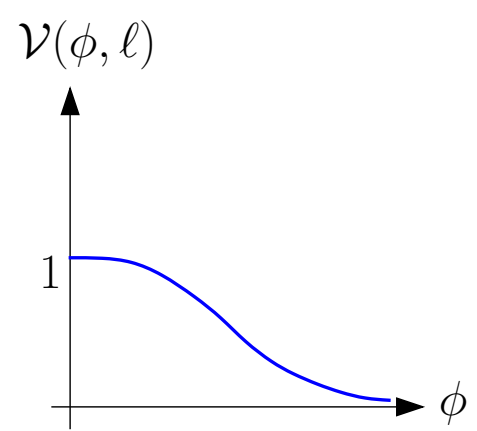

(b)

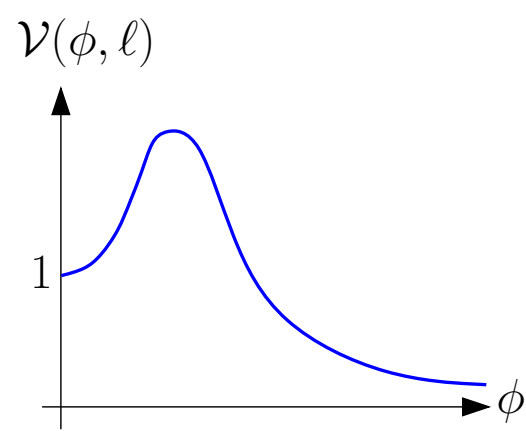

(c)

Figure 1: The D-branes anti-D-brane potential. a): For non-zero tachyon field, $\phi$, potential has one minimum in the $\ell$ direction. b) For brane separation $\ell<$ $\sqrt{2 \pi^{2} \alpha^{\prime}}$, the potential has one maximum and one minimum in the $\phi$ direction. In this case, there is a classical homogeneous tachyon rolling solution. c): For brane separation $\ell>\sqrt{2 \pi^{2} \alpha^{\prime}}$, the potential has two minimums in the $\phi$ direction. In this case, there is non-perturbative tachyon tunneling solution. The effective potential is symmetric under $\phi \rightarrow-\phi$ and $\ell \rightarrow-\ell$.

the true vacuum. Using the Euclidean continuation of the following effective action

$$
S=-2 \int d^{p+1} \sigma \mathcal{V}(\phi, \ell) \sqrt{-\operatorname{det}\left(\eta_{a b}+\frac{1}{1+\frac{\phi^{2} \ell^{2}}{2 \pi \alpha^{\prime}}}\left(\frac{1}{4} \partial_{a} \ell \partial_{b} \ell+2 \pi \alpha^{\prime} \partial_{a} \phi \partial_{b} \phi\right)\right)},
$$

one would find the "bounce" solution and the rate for decaying $D_{p} \bar{D}_{p}$ to the closed string vacuum. Similar studies have been done in [20] using two-derivative truncation of the BSFT effective action. We will leave this calculation for the future works.

Finally, one may couple the $D_{3} \bar{D}_{3}$ system to FRW gravity, and studies its cosmological evolution. In the closed string channel and for $\ell>>\sqrt{2 \pi^{2} \alpha^{\prime}}$, this cosmology has been studied in [16. In this study, the motion of one of the branes in the gravity background produced by the other brane is considered as the cosmological evolution. In this case, with $\ell$ as inflaton, inflation occurs when branes are very far from each other for which there is no decay of branes to closed string vacuum. On the other hand in the open string channel, if initially the value of $\ell=0$, one may consider $\phi$ as inflaton. In this case, one would find the cosmology of the tachyon rolling [11]. A problem for tachyon inflation is that inflation occurs only when tachyon is around the top of its potential. This does not lead to enough number of e-folding. However, if initially the value of $\ell \sim \sqrt{2 \pi^{2} \alpha^{\prime}}$, the top of the tachyon potential becomes more flat. This increases the number of e-folding. For the case that the 
initial value of $\ell>\sqrt{2 \pi^{2} \alpha^{\prime}}$, even the tachyon tunneling may cause the inflation. In this case, the $D_{3} \bar{D}_{3}$ system experiences the old inflation scenario [28]. It would be interesting to study this cosmologies in more details.

Acknowledgement: I would like to thank K. Hashimoto for discussion. 


\section{References}

[1] M. Gutperle and A. Strominger, IHEP 0204,018 (2002) arXiv:hep-th/0202210; A. Sen, JHEP 0204, 048 (2002) arXiv:hep-th/0203211.

[2] A. Sen, JHEP 0207, 065 (2002) arXiv:hep-th/0203265.

[3] A. Sen, Mod. Phys. Lett. A 17, 1797 (2002) arXiv:hep-th/0204143.

[4] A. Sen, JHEP 0210, 003 (2002) arXiv:hep-th/0207105; A. Sen, arXiv:hep-th/0209122]; P. Mukhopadhyay and A. sen, JHEP 0211, 047 (2002) arXiv:hep-th/0208142 ; A. Strominger, arXiv:hep-th/0209090; F. Larsen, A. Naqvi and S. Terashima, JHEP 0302, 039 (2003) |arXiv:hep-th/0212248; M. Gutperle and A. Strominger, arXiv:hep-th/0301038; A. Maloney, A. Strominger and X. Yin, arXiv:hep-th/0302146; T. Okuda and S. Sugimoto, Nucl. Phys. B 647, 101 (2002) arXiv:hep-th/0208196; S.J. Rey and S. Sugimoto, arXiv:hep-th/0301049 S.J. Rey and S. Sugimoto, arXiv:hep-th/0303133.

[5] N. Lambert, H. Liu and J. Maldacena, "Closed strings from decaying D-branes," hep-th/0303139.

[6] A. Sen, arXiv:hep-th/0410103.

[7] A. Sen, HEP 9910, 008 (1999) arXiv:hep-th/9909062.

[8] M. R. Garousi, Nucl. Phys. B 584, 284 (2000) arXiv:hep-th/0003122.

[9] E. A. Bergshoeff, M. de Roo, T. C. de Wit, E. Eyras and S. Panda, JHEP 0005, 009 (2000) arXiv:hep-th/0003221.

[10] J. Kluson, Phys. Rev. D 62, 126003 (2000) hep-th/0004106.

[11] G. W. Gibbons, Phys. Lett. B 537, 1 (2002); M. Fairbairn and M. H. G. Tytgat, Phys. Lett. B 546, 1 (2002); A. Feinstein, Phys. Rev. D 66, 063511 (2002); S. Mukohyama, Phys. Rev. D 66, 024009 (2002); D. Choudhury, D. Ghoshal, D. P. Jatkar and S. Panda, Phys. Lett. B 544, 231 (2002); G. Shiu and I. Wasserman, Phys. Lett. B 541, 6 (2002); L. Kofman and A. Linde, JHEP 0207, 004 (2002); M. Sami, Mod. Phys. Lett. A 18, 691 (2003); A. Mazumdar, S. Panda and A. Perez-Lorenzana, Nucl. Phys. B 614, 101 (2001); J. c. Hwang and H. Noh, Phys. Rev. D 66, 084009 (2002); Y. S. Piao, R. G. Cai, X. m. Zhang and Y. Z. Zhang, Phys. Rev. D 66, 121301 (2002); J. M. Cline, H. Firouzjahi and P. Martineau, JHEP 0211, 041 (2002); G. N. Felder, L. Kofman and A. Starobinsky, JHEP 0209, 026 (2002); S. Mukohyama, Phys. Rev. D 66, 123512 (2002); M. Sami, P. Chingangbam and T. Qureshi, Phys. Rev. D 66, 043530 (2002); M. C. Bento, O. Bertolami and A. A. Sen, Phys. Rev. D 67, 063511 (2003); J. g. Hao and X. z. Li, Phys. Rev. D 66, 087301 (2002); 
C. j. Kim, H. B. Kim and Y. b. Kim, Phys. Lett. B 552, 111 (2003); T. Matsuda, Phys. Rev. D 67, 083519 (2003); A. Das and A. DeBenedictis, arXiv:gr-qc/0304017; Z. K. Guo, Y. S. Piao, R. G. Cai and Y. Z. Zhang, Phys. Rev. D 68, 043508 (2003); G. W. Gibbons, Class. Quant. Grav. 20, S321 (2003); M. Majumdar and A. C. Davis, arXiv:hep-th/0304226, S. Nojiri and S. D. Odintsov, Phys. Lett. B 571, 1 (2003); E. Elizalde, J. E. Lidsey, S. Nojiri and S. D. Odintsov, Phys. Lett. B 574, 1 (2003); D. A. Steer and F. Vernizzi, Phys. Rev. D 70, 043527 (2004); V. Gorini, A. Y. Kamenshchik, U. Moschella and V. Pasquier, Phys. Rev. D 69, 123512 (2004); L. P. Chimento, Phys. Rev. D 69, 123517 (2004); M. B. Causse, arXiv:astro-ph/0312206 B. C. Paul and M. Sami, Phys. Rev. D 70, 027301 (2004); G. N. Felder and L. Kofman, Phys. Rev. D 70, 046004 (2004); J. M. Aguirregabiria and R. Lazkoz, Mod. Phys. Lett. A 19, 927 (2004); L. R. Abramo, F. Finelli and T. S. Pereira, arXiv:astro-ph/0405041; G. Calcagni, arXiv:hep-th/0406006 J. Raeymaekers, JHEP 0410, 057 (2004); G. Calcagni and S. Tsujikawa, Phys. Rev. D 70, 103514 (2004) arXiv:astro-ph/0407543; M. R. Garousi, M. Sami and S. Tsujikawa, Phys. Rev. D 70, 043536 (2004); M. R. Garousi, M. Sami and S. Tsujikawa, Phys. Lett. B to appear arXiv:hep-th/0405012 ; S. K. Srivastava, arXiv:gr-qc/0409074 N. Barnaby and J. M. Cline, arXiv:hep-th/0410030 E.J. Copeland, M.R. Garousi, M. Sami and S. Tsujikawa, arXiv:hep-th/0411192.

[12] A. Sen, arXiv:hep-th/9904207.

[13] A. Sen, JHEP 9808, 010 (1998) arXiv:hep-th/9805019; S. De Alwis, Phys. Lett. B 461, 329 (1999) [ arXiv;hep-th/9905080|.

[14] S. Sen, Phys. Rev. D 68, 066008 (2003) arXiv:hep-th/0303057.

[15] P. Kraus and F. Larsen, Phys. Rev. D 63, 106004 (2001) arXiv:hep-th/0012198; T. Takayanagi, S. Terashima and T. Uesugi, JHEP 0103, 019 (2001) arXiv:hep-th/0012210; N. T. Jones and S.H. Tye, JHEP 0301, 012 (2003) arXiv:hep-th/0211180; D.P. Jatkar, G. Mandal and S.R. Wadia, JHEP 0009, 018 (2000) arXiv:hep-th/0007079; Y. Hikida, M. Nozaki and T. Takayanagi, Nucl. Phys. B 595, 319 (2001) arXiv:hep-th/0008023.

[16] G.R. Dvali and S.H.H. Tye, Phys. Lett. B 450, 72 (1999) arXiv:hep-ph/9812483; C.P. Burgess, M. Majumdar, D. Nolte, F. Quevedo, G. Rajesh and R.J. Zhang, JHEP 0107, 047 (2001) arXiv:hep-th/0105204; G.R. Dvali, Q. Shafi and S. Solganik, arXiv:hep-th/0105203; S. Kachru, R. Kallosh, A. Linde, J. Maldacena, L. McAllister and S. P. Trivedi, JCAP 0310, 013 (2003) arXiv:hep-th/0308055.

[17] M.R. Garousi, Nucl. Phys. B 647, 117 (2002) arXiv:hep-th/0209068; JHEP 0304, 027 (2003) arXiv:hep-th/0303239; JHEP 0305, 058 (2003) arXiv:hep-th/0304145; JHEP 0312, 036 (2003) arXiv:hep-th/0307197.

[18] J. Polchinski, "String Theory", Vol II, Cambridge (1998). 
[19] C.G. Callen and J.M. Maldacena, Nucl. Phys. B 513, 198 (1998) arXiv:hep-th/9708147.

[20] K. Hashimoto, JHEP 0207, 035 (2002) arXiv:hep-th/0204203.

[21] T. Banks and L. Susskind, arXiv:hep-th/9511194.

[22] A. Sen, JHEP 9812, 021 (1998) arXiv:hep-th/9812031; P. Horava, Adv. Theor. Math. 2, 1373 (1999) arXiv:hep-th/9812135.

[23] M. Alishahiha, H. Ita and Y. Oz, Phys. Lett. B 503, 181 (2001) arXiv:hep-th/0012222; N.D. Lambert and I. Sachs, Phys. Rev. D 67, 026005 (2003) arXiv:hep-th/0208217, Y. Kim, O.K. Kwon and C.O. Lee, arXiv:hep-th/0411164.

[24] C.J. Kim, H.B. Kim, Y.B. Kim and O.K. Kwon, JHEP 0303, 008 (2003) arXiv:hep-th/0301076; F. Leblond and A.W. Peet, JHEP 0304, 048 (2003) arXiv:hep-th/0303035.

[25] B. Janssen and P. Meessen, Phys. Lett. B 526, 144 (2002) [ arXiv:hep-th/0009025.

[26] M. R. Garousi and R. C. Myers, Nucl. Phys. B 542, 73 (1999) [raXiv:hep-th/9809100.

[27] S. Coleman, Phys. Rev. D 15, 2929 (1977).

[28] A.H. Guth, Phys. Rev. D 23, 347 (1981). 